DYNAMICAL SYSTEMS AAND ERGODIC THEORY BANACH CENTER PUBLICATIONS, VOLUME 23 PWN - POLISH SCIENTIFIC PUBLISHERS WARSZAWA 1989

\title{
APPROXIMATING ENTROPY OF MAPS OF THE INTERVAL
}

\author{
LOUIS BLOCK \\ Department of Mathematics, University of Florida \\ Gainesville, FL U.S.A. \\ ETHAN M. COVEN \\ Department of Mathematics, Wesleyan University \\ Middletown, CT U.S.A.
}

This paper extends some results of M. Misiurewicz [M], Y. Takahashi [T], and L.-S. Young [Y], showing that the topological entropy of a continuous map of the interval is determined by its periodic orbits and its symbolic dynamics.

Let $f$ denote a continuous map of a compact interval $I$ to itself, and let $h$ ( ) denote topological entropy [AKM]. If $P=\left\{x_{1}<x_{2}<\ldots<x_{N}\right\}$ is a periodic orbit of $f$, let $L_{P}$ denote the "connect-the-dots" map determined by $P$, i.e., $L_{P}:\left[x_{1}, x_{N}\right] \rightarrow\left[x_{1}, x_{N}\right]$ agrees with $f$ on $P$, and is linear on each interval $\left[x_{i}, x_{i+1}\right]$.

THEOREM A. $h(f)=\sup h\left(L_{P}\right)$, where the supremum is taken over all periodic orbits $P$ of $f$.

Theorem A is due originally to Takahashi [T, Theorem 3], although his proof appears to be valid only for piecewise monotone maps (see MR $821: 58057)$.

Theorem B. Suppose $h(f)>0$, and let $\varepsilon>0$. There is a collection $K_{1}, \ldots, K_{n}$ of pairwise disjoint closed subintervals of I such that $\log \lambda \geqslant h(f)$ $-\varepsilon$, where $\lambda$ is the largest eigenvalue of the $n \times n$ matrix $B$ defined by $B_{i j}=1$ if $f\left(K_{i}\right) \supseteq K_{j}$, and $B_{i j}=0$ otherwise.

This work was done while the second author was at the Institute for Advanced Study, Princeton, NJ. He thanks the Institute for its hospitality and support. 
In this case, there is a closed invariant subset $X$ (contained in the set of points whose orbits lie in $K_{1} \cup \ldots \cup K_{n}$ ) such that $f \mid X$ is semiconjugate to $S$, the shift of finite type associated to the matrix $B$. Since $h(f \mid X) \geqslant h(S)$ $=\log \lambda$, we may approximate the topological entropy of $f$ arbitrarily well from its symbolic dynamics. For piecewise monotone $f$, Young [Y, Lemma 2.7] showed that given $\varepsilon>0$, there is a closed invariant set $X$ such that $h(f \mid X) \geqslant h(f)-\varepsilon$ and $f \mid X$ is actually conjugate to a shift of finite type.

Our proofs of both theorems are based on the following theorem of Misiurewicz.

THEOREM 1 [M, Theorem 1]. Let $\varepsilon>0$. There are positive integers $n$ and $d$, a subinterval $J$ of $I$, and subintervals $J_{1}, \ldots, J_{d}$ of $J$ with pairwise disjoint interiors, such that $f^{n}\left(J_{i}\right) \supseteq J$ for $i=1, \ldots, d$, and $(1 / n) \log d \geqslant h(f)-\varepsilon$.

(By shrinking $J$ a bit, we can choose $J_{1}, \ldots, J_{d}$ to be pairwise disjoint, thus obtaining symbolic dynamics for $f^{n}$.)

We also use the following corollary of Theorem 1.

Theorem 2 [M, Theorem 2]. Topological entropy, regarded as a function on the space of all continuous maps from I to itself, is lower semi-continuous.

We will use the following two lemmas. The first follows from [BGMY, Lemma 1.5].

LeMma 3. If $P$ is a periodic orbit of $f$, then $h(f) \geqslant h\left(L_{P}\right)$.

LEMMA 4. If $P$ is a periodic orbit of $f$ and $Q \subseteq P$ is a periodic orbit of $f^{n}$, then $h\left(L_{P}\right) \geqslant(1 / n) h\left(L_{Q}\right)$.

Proof. Since $Q$ is a periodic orbit of $L_{P}^{n}$, it follows from Lemma 3 that $h\left(L_{P}^{n}\right) \geqslant h\left(L_{Q}\right)$. But $h\left(L_{P}^{n}\right)=n h\left(L_{P}\right)$ [AKM, Theorem 2].

We will associate to a periodic orbit a Markov graph and a type. Let $P$ $=\left\{x_{1}<x_{2}<\ldots<x_{N}\right\}$ be a periodic orbit of $f$. Let $I_{1}=\left[x_{1}, x_{2}\right], \ldots, I_{N-1}$ $=\left[x_{N-1}, x_{N}\right]$. The Markov graph associated to $P$ is the directed graph with vertices $I_{1}, \ldots, I_{N-1}$, and an edge (arrow) from $I_{i}$ to $I_{j}$ if and only if $L_{P}\left(I_{i}\right) \supseteq I_{j}$. Given a loop in the graph, there is a periodic orbit of $f$ which goes through the vertices in the prescribed order. The type of $P$ is the permutation $\pi$ of $\{1, \ldots, N\}$ defined by $\pi(i)=j$ if $f\left(x_{i}\right)=x_{j}$. Then $h\left(L_{P}\right)$ is the logarithm of the largest eigenvalue of the adjacency matrix of the Markov graph associated to $P$, and in particular, if $P$ and $Q$ have the same type, then $h\left(L_{p}\right)=h\left(L_{Q}\right)$.

We may also form a Markov graph using any finite set of points in place of $P$. In this case too, loops in the graph yield periodic orbits of $f$.

Proof of Theroem A. It follows from Lemma 3 that $h(f) \geqslant \sup h\left(L_{P}\right)$. To obtain the reverse inequality, it suffices to prove the following. 
(*) For any $\varepsilon>0$ and any positive integer $M$, there is a periodic orbit $P$ of $f$, containing at least $M$ points, such that $h\left(L_{P}\right) \geqslant h(f)-\varepsilon$.

(The condition that $P$ have at least $M$ points will be used in the proof of Theorem B, but not in the proof of Theorem A.)

Let $\varepsilon$ and $M$ be given. By Theorem 1, there are positive integers $n$ and $d_{\text {, }}$ a subinterval $J$ of $I$, and subintervals $J_{1}, \ldots, J_{d}$ of $J$ with pairwise disjoint interiors, such that $f^{n}\left(J_{i}\right) \supseteq J$ for $i=1, \ldots, d$, and $(1 / n) \log d \geqslant h(f)-\varepsilon / 2$. We may assume that $J$ and $J_{1}, \ldots, J_{d}$ are closed (by taking closures if necessary).

We introduce a piecewise linear map $L$, which will serve as a model for the relevant dynamics of $f^{n}$ on $J$. Let $J=[a, b]$. There are points $w_{0}<w_{1}$ $<\ldots<w_{d}$ in $J$ such that either

(1) $f^{n}\left(w_{i}\right)=a$ for $i$ even, and $f^{n}\left(w_{i}\right)=b$ for $i$ odd, or

(2) $f^{n}\left(w_{i}\right)=a$ for $i$ odd, and $f^{n}\left(w_{i}\right)=b$ for $i$ even.

Without loss of generality, we assume the former.

Let $L$ be the piecewise linear map of $[0,1]$ to itself satisfying

(3) $L(0)=0, L(1 / d)=1, L(2 / d)=0, L(3 / d)=1, \ldots$,

and

(4) The restriction of $L$ to the intervals $[0,1 / d], \ldots,[(d-1) / d, 1]$ is linear.

Note that $h(L)=\log d$.

We first claim that given $\delta>0$, there is a periodic orbit of $L$ which meets every subinterval of $[0,1]$ of length $\delta$. To see this, take a finite open cover of $[0,1]$ by intervals of length less than $\delta / 2$. There is a positive integer $m$ such that $L^{m}(C)=[0,1]$ for every $C$ in this cover. Therefore, in the Markov graph associated to the set of endpoints of the members of the cover, there is a loop which passes through every vertex, and hence there is a periodic orbit of $L$ which meets every member of the cover. But any interval of length $\delta$ contains a member of the cover.

Choose $\delta$ small enough so that the periodic orbit $R$ described above contains at least $M$ points. Extend $L_{R}$ to a map $\bar{L}_{R}$ of $[0,1]$ to itself by defining $\bar{L}_{R}$ to be constant on $[0, \min R]$ and $[\max R, 1]$. Then $h\left(\bar{L}_{R}\right)$ $=h\left(L_{R}\right)$ and $\bar{L}_{R}$ is within $d \delta$ of $L$ in the $C^{0}$-metric. By Theorem 2, we may choose $\delta$ small enough so that $h\left(L_{R}\right) \geqslant \log d-\varepsilon / 2$.

We next claim that $f^{n}$ has a periodic orbit $Q$ of the same type as $R$. To prove this, we define a nested sequence $\left\{A_{k}\right\}$ of finite sets, by

(5) $A_{0}=\{0,1 / d, 2 / d, \ldots,(d-1) / d, 1\}$,

(6) $A_{k}=A_{k-1} \cup L^{-1}\left(A_{k-1}\right), k \geqslant 1$. 
Since $A_{k}=\left\{j / d^{k+1} \mid 0 \leqslant j \leqslant d^{k+1}\right\}$, it follows that $\bigcup_{0 \leqslant k<\infty} A_{k}$ is dense, and so there is a positive integer $k$ such that any interval joining adjacent points of $A_{k}$ contains at most one point of $R$.

Let $B_{0}=\left\{w_{0}, \ldots, w_{d}\right\}$. Note that $B_{0}$ and $A_{0}$ have the same number of points. Choose a subset $B_{1}$ of $B_{0} \cup f^{-n}\left(B_{0}\right)$ such that

(7) $B_{1} \supseteq B_{0}$,

(8) $B_{1}$ and $A_{1}$ have the same number of points,

(9) If $A_{1}=\left\{y_{0}<\ldots<y_{m}\right\}$ and $B_{1}=\left\{z_{0}<\ldots<z_{m}\right\}$, then $y_{i} \in A_{0}$ if and only if $z_{i} \in B_{0}$, and for $y_{i} \in A_{1}-A_{0}, L\left(y_{i}\right)=y_{j}$ if and only if $f^{n}\left(z_{i}\right)=z_{j}$.

We continue inductively, obtaining $B_{2}, \ldots, B_{k}$ satisfying the appropriate analogous conditions. Then, the Markov graph determined by the points of $A_{k}$ and $L$ can be embedded in the Markov graph determined by the points of $B_{k}$ and $f^{n}$.

There is a loop in the Markov graph determined by the points of $A_{k}$ and $L$ which corresponds to the periodic orbit $R$. Hence the same loop appears in the Markov graph determined by the points of $B_{k}$ and $f^{n}$. This loop yields a periodic orbit $Q$ of $f^{n}$ which has the same type as $R$. The claim is established.

Let $P$ be the unique periodic orbit of $f$ which contains $Q$. By Lemma 4 , $h\left(L_{P}\right) \geqslant(1 / n) h\left(L_{Q}\right)$. Thus

$$
h\left(L_{P}\right) \geqslant(1 / n)(\log d-\varepsilon / 2) \geqslant(1 / n) \log d-\varepsilon / 2 \geqslant h(f)-\varepsilon .
$$

This proves (*) and hence Theorem A.

Proof of Theorem B. By Theorem A, there is a periodic orbit $R$ of $f$ such that $h\left(L_{R}\right) \geqslant h(f)-\varepsilon / 2$. It follows from (*) above, applied to $L_{R}$, that $L_{R}$ has a periodic orbit $Q=\left\{q_{1}<\ldots<q_{N}\right\}$, with $N>2, Q \neq R$ (and hence $Q \cap R=\emptyset$ ), and $h\left(L_{Q}\right) \geqslant h\left(L_{R}\right)-\varepsilon / 2$.

We will first find a collection of pairwise disjoint closed intervals $J_{i} \subseteq\left[q_{i}, q_{i+1}\right], i=1, \ldots, N-1$, such that if $L_{Q}\left[q_{i}, q_{i+1}\right] \supseteq\left[q_{j}, q_{j+1}\right]$, then $L_{R}\left(J_{i}\right) \supseteq J_{j}$.

Since $N>2$, there is an integer $n, 1<n<M$, such that $L_{R}\left(q_{n}\right)=q_{1}$ or $q_{N}$. Relabel $Q$ as $\left\{\alpha_{1}, \ldots, \alpha_{N}\right\}$, where $\alpha_{1}=q_{n}$, and $L_{R}\left(\alpha_{i}\right)=\alpha_{i-1}$ for $i$ $=2, \ldots, N$. Note that $L_{R}$ is strictly monotone at each $\alpha_{i}$. Let $\sigma_{0}=+1$ or -1 according as $L_{R}\left(q_{n}\right)=q_{N}$ or $q_{1}$, and for $i=1, \ldots, N$, let $\sigma_{i}=+1$ or -1 according as $L_{R}$ is increasing or decreasing at $\alpha_{i}$. Finally, let $\tau_{i}=\sigma_{0} \sigma_{1} \ldots \sigma_{i}$. Then $L_{R}$ maps the $\tau_{1}$ side of $\alpha_{1}$ outside of $\left[q_{1}, q_{N}\right]$, and for $i=2, \ldots, N$, it maps the $\tau_{i}$ side of $\alpha_{i}$ to the $\tau_{i-1}$ side of $\alpha_{i-1}$.

We define a collection of points $\beta_{1}, \ldots, \beta_{N}$, inductively, as follows. If $\tau_{1}$ $=-1$, let $\beta_{1}$ be the largest point in $L_{R}^{-1}(R)$ which is less than $\alpha_{1}$, and if $\tau_{1}$ $=+1$, let $\beta_{1}$ be the smallest point in $L_{R}^{-1}(R)$ which is greater than $\alpha_{1}$. 
Assuming that $\beta_{1}, \ldots, \beta_{i-1}$ have been defined, if $\tau_{i}=-1$, let $\beta_{i}$ be the largest point in $L_{R}^{-1}\left(\beta_{i-1}\right)$ which is less than $\alpha_{i}$, and if $\tau_{i}=+1$, let $\beta_{i}$ be the smallest point in $L_{R}^{-1}\left(\beta_{i-1}\right)$ which is greater than $\alpha_{i}$.

Note that $L_{R}\left(\beta_{1}\right) \notin\left[q_{1}, q_{N}\right]$, and that $\left\langle\alpha_{i}, \beta_{i}\right\rangle \cap Q=\varnothing$ for $i=1, \ldots, N$, where $\left\langle\alpha_{i}, \beta_{i}\right\rangle$ denotes the open interval with endpoints $\alpha_{i}$ and $\beta_{i}$. Also, $L_{R}\left\langle\alpha_{1}, \beta_{1}\right\rangle \cap\left[q_{1}, q_{N}\right]=\emptyset$ and $L_{R}\left\langle\alpha_{i}, \beta_{i}\right\rangle=\left\langle\alpha_{i-1}, \beta_{i-1}\right\rangle$ for $i=2, \ldots, N^{\prime}$. Finally, if $L_{Q}\left[q_{i}, q_{i+1}\right] \supseteq\left[q_{j}, q_{j+1}\right]$, then

and

$$
L_{R}\left(\left[q_{i}, q_{i+1}\right]-\left\langle\alpha_{1}, \beta_{1}\right\rangle\right) \supseteq\left[q_{j}, q_{j+1}\right]
$$

$L_{R}\left(\left[q_{i}, q_{i+1}\right]-\left\langle\alpha_{k}, \beta_{k}\right\rangle\right) \supseteq\left[q_{j}, q_{j+1}\right]-\left\langle\alpha_{k-1}, \beta_{k-1}\right\rangle, \quad k=2, \ldots, N$.

For $i=1, \ldots, N-1, \quad$ let $J_{i}=\left[q_{i}, q_{i+1}\right]-\bigcup_{1 \leqslant j \leqslant N}\left\langle\alpha_{j}, \alpha_{j}\right\rangle$. Then $J_{1}, \ldots, J_{N-1}$ is the desired collection of pairwise disjoint closed intervals such that $L_{R}\left(J_{i}\right) \supseteq J_{j}$ whenever $L_{Q}\left[q_{i}, q_{i+1}\right] \supseteq\left[q_{j}, q_{j+1}\right]$.

As in [B, Theorem 3.3], there is a periodic orbit $P$ of $f$ such that the action of $f$ on $P \cup R$ is the same as the action of $L_{R}$ on $Q \cup R$. By this we mean that $P$ and $Q$ have the same number of points, and if $P \cup R=\left\{x_{1}\right.$ $\left.<\ldots<x_{M}\right\}$ and $Q \cup R=\left\{y_{1}<\ldots<y_{M}\right\}$, then $f\left(x_{i}\right)=x_{j}$ if and only if $L_{R}\left(y_{i}\right)=y_{j}$. As in [B, Theorem 3.3] again, there are points $\gamma_{1}, \ldots, \gamma_{N}$, corresponding to $\beta_{1}, \ldots, \beta_{N}$, such that the action of $f$ on $P \cup R \cup\left\{\gamma_{1}, \ldots, \gamma_{N}\right\}$ is the same as the action of $L_{R}$ on $Q \cup R \cup\left\{\beta_{1}, \ldots, \beta_{N}\right\}$. Thus we obtain a collection of pairwise disjoint closed intervals $K_{1}, \ldots, K_{N-1}$ (defined analogously to $\left.J_{1}, \ldots, J_{N-1}\right)$, such that $f\left(K_{i}\right) \supseteq K_{j}$ whenever $L_{Q}\left[q_{i}, q_{i+1}\right]$ $\supseteq\left[q_{j}, q_{j+1}\right]$.

Finally, let $A$ be the adjacency matrix of the Markov graph associated to $Q$, and let $\mu$ denote the largest eigenvalue of $A$. Let $B$ and $\lambda$ be as in the statement of the theorem. Since $B_{i j}=1$ whenever $A_{i j}=1$, we have $\log \lambda$ $\geqslant \log \mu=h\left(L_{Q}\right) \geqslant h(f)-\varepsilon$. This completes the proof of the theorem.

\section{References}

[AKM] R. L. Adler, A. G. Konheim and M. H. McAndrew, Topological entropy, Trans. Amer. Math. Soc. 114 (1965), 309-319.

[B] - S. Baldwin, Generalizations of a theorem of Sarkovskii on orbits of continuous real-valued functions, Discrete Math. 67 (1987), 111-127.

[BGMY] L. Block, J. Guckenheimer, M. Misiurewicz, and L.-S. Young, Periodic points and topological entropy of one-dimensional maps, Springer Lectures Notes in Math. 819, 18-34, 1980.

[M] M. Misiurewicz, Horseshoes for mappings of the interval, Bull. Acad. Polon. Sci. Ser. Sci. Math. 27 (1979), 167-169.

[T] Y. Takahashi, A formula for topological entropy of one-dimensional dynamics, Sci. Papers Coll. Gen. Ed. Univ. Tokyo 30 (1980), 11-22.

[Y] L.-S. Young, On the prevalence of horseshoes, Trans. Amer. Math. Soc. 263 (1981), 75-88. 